\title{
Incidence and risk factors for diabetic retinopathy in the communities of Shenzhen
}

\author{
Yun Peng ${ }^{1}$, Xianxian Guo ${ }^{2}$, Junan Liu ${ }^{2}$, Yuanyuan Yao ${ }^{1}$, Hui Guo ${ }^{1}$, Yuan Wang ${ }^{1}$ Xuan Xiao ${ }^{3}$, in Ye \\ ${ }^{1}$ Shenzhen Eye Hospital, Shenzhen Key Laboratory of Ophthalmology, Affiliated Shenzhen Eye Hospital of Jinan University, Shenzhen, China; \\ ${ }^{2}$ School of Public Health, Tongji Medical College of Huazhong University of Science Technology, Wuhan, China; ${ }^{3}$ Eye Center, Renmin Hospital of \\ Wuhan University, Wuhan, China \\ Contributions: (I) Conception and design: L Ye, Y Peng; (II) Administrative support: L Ye; (III) Provision of study materials or patients: Y Peng; \\ (IV) Collection and assembly of data: Q Guo, J Liu; (V) Data analysis and interpretation: Q Guo; (VI) Manuscript writing: All authors; (VII) Final \\ approval of manuscript: All authors. \\ Correspondence to: Lin Ye. Department of Ophthalmoplastic Lacrimal Apparatus, Shenzhen Eye Hospital, 18 Fengtian Road, Shenzhen 518040, \\ China. Email: yelin0711@126.com; Xuan Xiao. Eye Center, Renmin Hospital of Wuhan University, 238 Jiefang Road, Wuhan 430061, China. \\ Email: xiaoxuan1111@163.com.
}

Background To understand the prevalence of diabetic retinopathy (DR) in Shenzhen and to analyze the risk factors for the occurrence and development of DR. Based on the comprehensive information system for diabetes prevention and control in the communities of Shenzhen in 2019, six community health service centers in Shenzhen were used as research sites to carry out multicenter, cross-sectional screening studies.

Methods: Cluster random sampling was used to collect data from 904 patients with diabetes in Shenzhen. The occurrence of DR and vision-threatening diabetic retinopathy (VTDR) was analyzed, and multivariate logistic regression was performed to analyze the risk factors for DR and VTDR. EpiData version 3.1 (EpiData Association, Odense, Denmark) statistical software was used to build a database, and Statistical Package for the Social Sciences (SPSS) version 25.0 (IBM Corp., Armonk, N.Y., USA) was used to sort up and analyze the data.

Results: The prevalence of DR among diabetic patients in Shenzhen was 18.58\% [95\% confidence interval (CI): $16.13-21.3 \%]$, and the prevalence of VTDR was $2.43 \%$ (95\% CI: $1.57-1.2 \%$ ). The prevalence of DR and VTDR was higher in males than in females. Logistic regression analysis showed that age, disease duration, medication mode, and the occurrence of diabetic peripheral neuropathy (DPN) were associated with the incidence of DR in diabetic patients, and that disease duration, the occurrence of DPN, and diabetic nephropathy were associated with the occurrence of VTDR.

Conclusions: The prevalence of DR in the communities of Shenzhen is high. Age, disease duration, medications, and DPN are the major risk factors for the occurrence of DR.

Keywords: Diabetic retinopathy (DR); vision-threatening diabetic retinopathy (VTDR); community screening; risk factors

Submitted Nov 06, 2020. Accepted for publication Jan 22, 2021.

doi: 10.21037/apm-20-2526

View this article at: http://dx.doi.org/10.21037/apm-20-2526

\section{Introduction}

Over the past 30 years, the prevalence of diabetes in China has rapidly increased, and the incidence of diabetic retinopathy (DR) has also gradually increased (1). In 2012, a meta-analysis based on 35 epidemiological studies worldwide showed that
$34.6 \%$ of diabetic patients had DR and that $10.2 \%$ of diabetic patients had vision-threatening DR (VTDR). That is, there are approximately 93 million DR and VTDR patients worldwide, resulting in tremendous public health issues globally $(2,3)$. Recently, epidemiological studies have been conducted on 
the prevalence of, risk factors for, and progression of DR in countries such as the United States (4), England (5-7), China $(8,9)$, Portugal $(10)$ and so on $(11,12)$. In 1998, Shenzhen initiated comprehensive prevention and treatment work for hypertension and diabetes in the community, emerging as the first group of cities in China to become a "National Demonstration Site for Comprehensive Prevention and Control of Chronic Diseases" and a "Demonstration City of Comprehensive Prevention and Control of Chronic Diseases in Guangdong Province". However, at this stage, each community health service center lacks disease management for DR patients. The present research relies on community diabetes management in Shenzhen to understand the prevalence of DR and to analyze its influencing factors through community screening. This study aims to provide a basis for improving the intervention management of DR in communities in China.

We present the following article in accordance with the MDAR checklist (available at http://dx.doi.org/10.21037/ apm-20-2526).

\section{Methods}

Based on the comprehensive information system for diabetes prevention and control in the communities of Shenzhen in 2019, six community health service centers in Shenzhen were used as research sites to carry out multicenter, cross-sectional screening studies.

\section{Study subjects}

In this study, we conducted a survey using cluster random sampling and used type II diabetic patients registered at the Shenzhen Community Health Service Center as study subjects. The inclusion criteria for study subjects were as follows: (I) diabetic patients aged over 20 years; and (II) healthy patients who could receive routine DR screening. Patients were excluded based on the following criteria: (I) patients with ketoacidosis, hyperosmolar diabetic acidosis, or other serious diseases; (II) patients with mental illness or severe cognitive impairment; (III) patients who were pregnant during the study; and (IV) patients who were from the town and unwilling to participate. This study was approved by the Ethics Committee of Shenzhen Eye Hospital (No. 2017041201), and informed consent was obtained from all diabetic patients who participated in the screening. The study was conducted in accordance with the Declaration of Helsinki (as revised in 2013).

\section{Sampling method}

At present, the prevalence rate of DR in the Chinese diabetic population is $25-35 \%$, and the prevalence rate of DR in the diabetic population of Shenzhen is approximately $25 \%$. According to the random sampling formula $\mathrm{n}=(\mathrm{Z} 1-\mathrm{a} / 2 / \delta)$ 2 p (1-p) (allowable error, 4\%; $\alpha=0.05$ ), n was approximately 550. Considering the errors caused by cluster sampling, approximately 825 diabetic patients were required, thereby increasing the calculated sample size (n) by $50 \%$. This study expanded the sample size of diabetic patients to 1,000 based on a compliance rate of $80 \%$. Finally, the data of 904 diabetic patients were collected, and the recovery rate was $90.4 \%$.

\section{Survey method}

\section{Appointments for diabetic patients}

Diabetic patients' information was obtained through the community comprehensive prevention and treatment information system for diabetic patients in Shenzhen. Diabetic patients were regularly scheduled (via text message or telephone conversation) to attend the community health center (where health records are located) for free retinal screening.

\section{Questionnaire}

Relevant personnel were trained to administer the self-made "Shenzhen Diabetic Retinopathy Screening Questionnaire" in face-to-face interviews. The general information included patient's sex, age, education level, occupation, smoking and drinking history, exercise history, diet, family history of diabetes, duration of diabetes, medication status, and disease history, among other information.

\section{Fundus examinations}

In the community health service center, a trained general practitioner performed fundus examinations using a handheld ophthalmoscope to observe the general conditions of the anterior segment of the eye. Shenzhen Eye Hospital has been regularly sitting in many social health centers in the city to coach general practitioners for fundus examination and fundus photography training for 5 years before. Before the formal start of the project, the ophthalmologists in the members of our group have again trained and assessed the general practitioners of the social health center selected in the project. The ophthalmologist of our hospital carries on the remote interpretation to the fundus photography image data and the comprehensive diagnosis analysis to each screening patient all screening data. Patients with DR were preliminarily selected. 
Table 1 Basic information of the study subjects

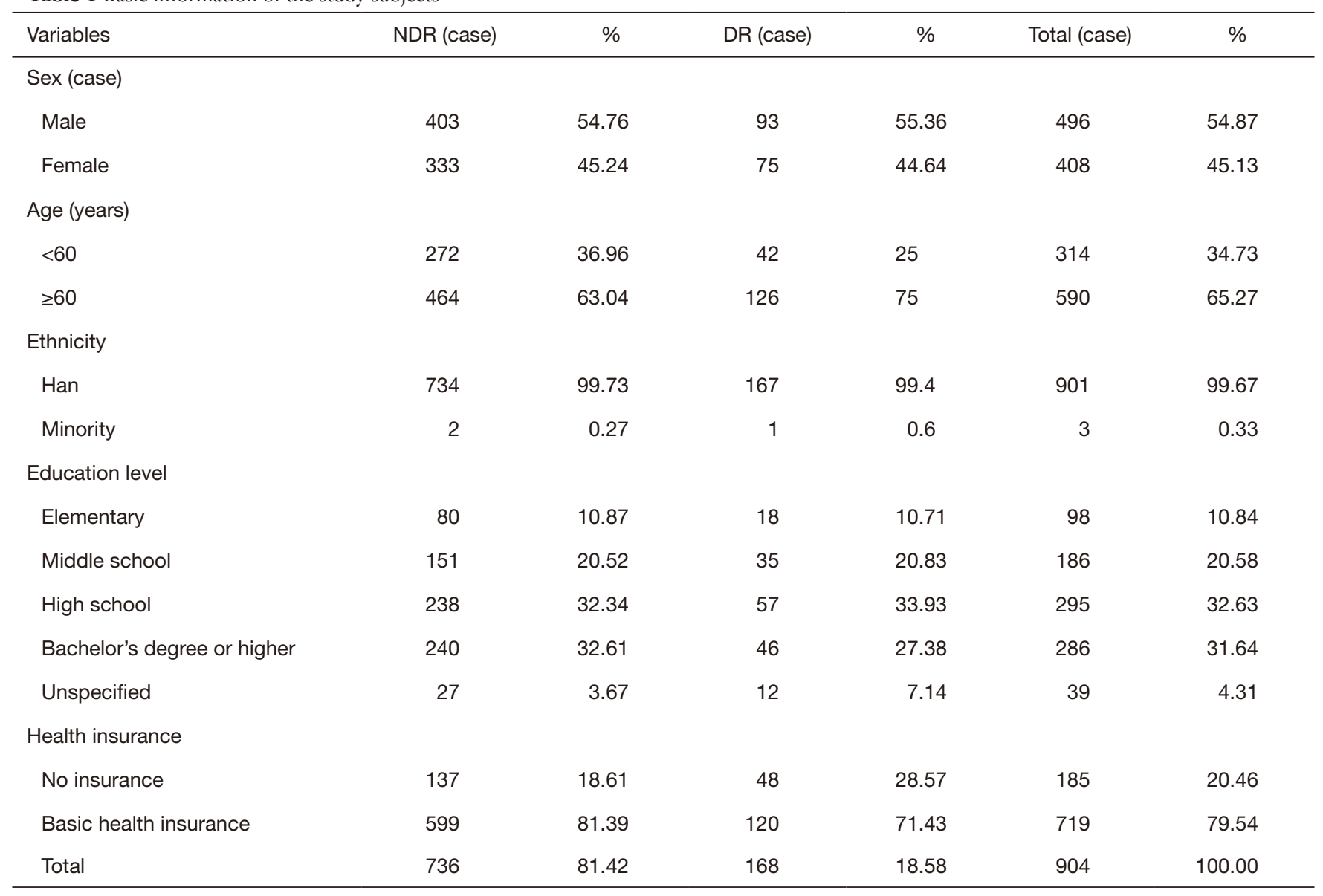

NDR, non-diabetic retinopathy; DR, diabetic retinopathy.

High-risk DR patients were informed and transferred to Shenzhen Eye Hospital, where a digital retinal exam without dilation was conducted. Two 35-degree fundus posterior pole images were taken, and fundus fluorescein angiography was performed. Disease stage (stages $0-\mathrm{IV}$ ) was determined in accordance with 2003 International Classification of DR (13). based on the diagnosis results. VTDR includes severe non proliferative DR (NPDR), proliferative DR (PDR), and clinically significant macular edema (14).

\section{Statistical analysis}

EpiData version 3.1 (EpiData Association, Odense, Denmark) statistical software was used to build a database, and Statistical Package for the Social Sciences (SPSS) version 25.0 (IBM Corp., Armonk, N.Y., USA) was used to sort up and analyze the data. Measurement data with a normal distribution were presented as the mean \pm standard deviation (SD). A t test or analysis of variance (ANOVA) was used for intergroup comparisons. Count data were presented as relative numbers, and the chi-square test was used for intergroup comparisons. Multivariate logistic regression analysis was performed to identify risk factors. The results were expressed as odds ratio (OR) [95\% confidence interval (CI)]. $\alpha=0.05$ was used as the threshold for significance for all statistical tests.

\section{Results}

\section{Basic information}

A total of 904 patients [ 496 males (54.87\%) and 408 females $(45.13 \%)]$ were enrolled in this study, including 736 nondiabetic retinopathy (NDR) patients $(81.42 \%)$ and 168 DR patients (18.58\%). The average age of the patients was $63.07 \pm 11.28$ years (Table 1 ). 


\section{Prevalence of DR}

Of the 904 diabetic patients enrolled in this study, 168 were DR patients (112 patients with mild NPDR, 34 patients with moderate PDR, 17 patients with severe PDR, five patients with PDR, and 22 patients with VTDR). Among the DR patients, 93 were male $(55.36 \%)$, and 75 were female $(44.64 \%)$, with an average age of $65.13 \pm 9.73$ years. The prevalence of DR was $18.58 \%$ (95\% CI: $16.13-21.3 \%$ ), of which, the prevalence rates for NPDR, PDR, and VTDR were $18.03 \%$ (95\% CI: $15.611-2.73 \%), 0.55 \%$ (95\% CI: $0.2-1.36 \%$ ), and $2.43 \%$ (95\% CI: $1.57-3.72 \%$ ), respectively. The prevalence of DR was higher in males than in females.

\section{Differential analysis of risk factors for DR and VTDR}

Differential analysis of the risk factors for DR and VTDR was performed in terms of biological factors, disease history, and behavioral factors. The prevalence of DR was significantly higher in patients with the following characteristics: age $\geq 60$ years, longer disease duration, the use of hypoglycemic drugs (HDs), diabetic foot, and diabetic peripheral neuropathy (DPN) $(\mathrm{P}<0.05$, Table 2). The prevalence of VTDR was markedly higher in patients with the following characteristics: longer disease duration, HDs, DPN, and diabetic nephropathy $(\mathrm{P}<0.05)$. Because of the limited number of cases collected by this epidemiological study and limited by project funds, we can't draw the conclusion that a significant increase in the prevalence also indicate an increase in the prevalence of severe PDR.

\section{Multivariate logistic regression analysis}

Dimensional indices, such as demographic characteristics, biological indicators, disease history, and behavioral factors, were used as independent variables, while DR or VTDR were used as the dependent variables. $\mathrm{P}<0.05$ was used as the inclusion criterion and $\mathrm{P}>0.1$ was used as the exclusion criterion. Multivariate logistic stepwise regression analysis was performed. The results indicated that the risk factors for DR included the type of health insurance $(\mathrm{OR}=0.637$; basic health insurance $v s$. no insurance; $\mathrm{P}=0.028$ ), duration of diabetes $(\mathrm{OR}=2.324 ; \geq 15$ vs. $<5$ years; $\mathrm{P}=0.001)$, medication $(\mathrm{OR}=6.070 ; \mathrm{HD}+$ insulin $v s$. no medication, diet + exercise; $\mathrm{P}=0.001 / \mathrm{OR}=3.334$; oral HD administration only $v$. no medication, diet + exercise; $\mathrm{P}=0.011)$, and $\mathrm{DPN}(\mathrm{OR}$ =3.042; with DPN vs. without DPN; $\mathrm{P}=0.004$ ) (Table 3). The risk factors for VTDR included duration of diabetes (OR
$=23.645 ; \geq 15$ vs. $<5$ years; $\mathrm{P}=0.003)$, DPN (OR =7.100; with DPN vs. without DPN; $\mathrm{P}=0.001$ ), and diabetic nephropathy $(\mathrm{OR}=12.674$; with $v$ s. without; $\mathrm{P}=0.006$ ) (Table 3).

\section{Discussion}

This study showed that the prevalence of DR among diabetic patients in the communities of Shenzhen was $18.58 \%$ (95\% CI: $16.13-21.3 \%)$ and that the prevalence of VTDR was $2.43 \%$ (95\% CI: $1.57-1.2 \%$ ). Pan et al. investigated the prevalence of DR among diabetic patients in the communities of Suzhou and reported a prevalence rate of $18.00 \%$ (95\% CI: $15.50-20.60 \%)(14)$. The lifestyle can significantly reduce the prevalence of VTDR including pay attention to standardized management of blood glucose, control blood glucose level well, exercise regularly, regulate diet, quit smoking and alcohol. Song et al. conducted a meta-analysis in 2018, based on 31 published studies, on the prevalence of DR among the diabetic population and identified a prevalence rate of $18.45 \%$ (95\% CI: $14.77-22.43 \%$ ) (15). Our findings are consistent with the results of the aforementioned studies. However, our results were slightly lower than those reported by $\mathrm{Xu}$ et al. (prevalence rate of $24.70 \%$; $95 \%$ CI: 22.80 $26.60 \%$ ), who conducted a study of the current situation among diabetic patients in the communities of Beijing (16), and were also lower than the findings of Varma et al.'s study of Chinese Americans (prevalence rate of $35.80 \%, 95 \% \mathrm{CI}$ : $32.10-39.60 \%$ ) (17). In our study, the prevalence rate for VTDR was lower than that reported by Pan et al. $(4.40 \%$, 95\% CI: $3.10-5.80 \%)$ (14), which may be related to lifestyle differences and the age of the study population.

This study also showed that the risk factors for DR development among diabetic patients in the communities of Shenzhen were disease duration, type of health insurance, medication, and DPN, while the risk factors associated with VTDR were disease duration, DPN, and diabetic nephropathy. Other risk factors (such as elevated glycated hemoglobin levels, smoking, drinking, BMI, and dyslipidemia) reported in previous studies were not found to be associated with DR or VTDR in our study.

The duration of diabetes in the DR group was longer than that in the NDR group, and the prevalence of DR was 1.324 times higher in diabetic patients with a disease duration of more than 15 years compared to patients with a disease duration of less than 5 years. This is consistent with the results reported by Dutra et al. (prevalence of DR was 1.07 times higher in patients with a disease duration of diabetes more than 15 years compared to patients with 
Table 2 Differential analysis of risk factors for DR and VTDR in diabetic patients

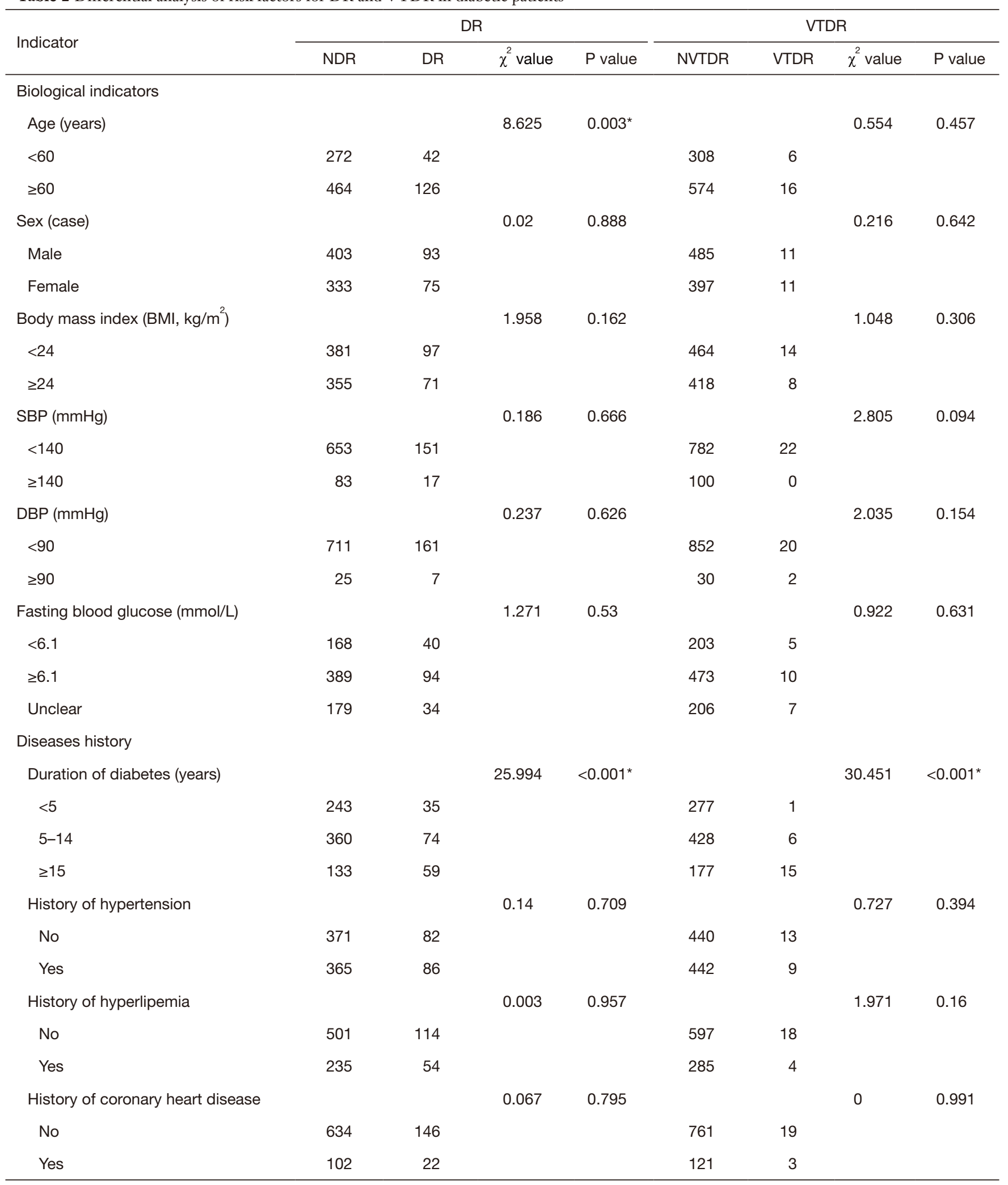

Table 2 (continued) 
Table 2 (continued)

\begin{tabular}{|c|c|c|c|c|c|c|c|c|}
\hline Indicator & \multicolumn{4}{|c|}{ DR } & \multicolumn{4}{|c|}{ VTDR } \\
\hline History of cerebral infarction & & & 0.355 & 0.551 & & & 0.001 & 0.978 \\
\hline No & 702 & 162 & & & 843 & 21 & & \\
\hline Yes & 34 & 6 & & & 39 & 1 & & \\
\hline No & 516 & 104 & & & 608 & 12 & & \\
\hline Yes & 12 & 7 & & & 18 & 1 & & \\
\hline Unclear & 208 & 57 & & & 256 & 9 & & \\
\hline DPN & & & 19.953 & $<0.001^{*}$ & & & 24.806 & $<0.001^{*}$ \\
\hline Unclear & 219 & 59 & & & 270 & 8 & & \\
\hline Diabetic nephropathy & & & 0.001 & 0.972 & & & 11.631 & $0.001^{*}$ \\
\hline No & 727 & 166 & & & 873 & 20 & & \\
\hline Yes & 9 & 2 & & & 9 & 2 & & \\
\hline \multicolumn{9}{|l|}{ Behavioral factors } \\
\hline \multicolumn{9}{|l|}{ Medication } \\
\hline Diet + exercise & 85 & 5 & & & 89 & 1 & & \\
\hline Insulin injection only & 44 & 10 & 28.595 & $<0.001^{*}$ & 50 & 4 & 9.851 & $0.020^{\star}$ \\
\hline Smoking & 122 & 26 & & & 147 & 1 & & \\
\hline Drinking status & & & 2.064 & 0.151 & & & 0.908 & 0.341 \\
\hline No drinking & 544 & 115 & & & 641 & 18 & & \\
\hline Drinking & 192 & 53 & & & 241 & 4 & & \\
\hline
\end{tabular}

* indicate the difference is significant. DR, diabetic retinopathy; VTDR, vision-threatening diabetic retinopathy; NDR, non-diabetic retinopathy; NVTDR, non-vision-threatening diabetic retinopathy.

a duration of diabetes less than 5 years) (18), and with those reported by Wat et al. and Raum et al. $(19,20)$. These findings are related to ocular fundus lesions caused by poor glycemic control. Diabetic patients usually have poor glycemic control, and thus, the longer the duration of diabetes, the more severe the damage to the structure and function of the ocular fundus, and the higher the prevalence of DR. The prevalence of DR and VTDR was higher in cases complicated by DPN, suggesting that diabetes damages the health of both large and small blood vessels. Although risk factors such as duration of diabetes, DPN, and diabetic nephropathy cannot be changed, these risk factors can help to determine the extent of screening for patients with DR. Individualized screening intervals for 
Table 3 Multivariate logistic regression analysis of retinopathy in diabetic patients in Shenzhen

\begin{tabular}{|c|c|c|c|c|c|c|}
\hline Indicator & $\mathrm{B}$ & SE & Wald & Sig & $\operatorname{Exp}(B)$ & $95 \% \mathrm{Cl}$ \\
\hline Constant & 2.389 & 0.614 & & & & \\
\hline \multicolumn{7}{|c|}{ Type of health insurance (control group: no insurance) } \\
\hline Basic health insurance & -0.451 & 0.205 & 4.848 & $0.028^{*}$ & 0.637 & $0.426-0.952$ \\
\hline $5-14$ years & 0.276 & 0.226 & 1.49 & 0.222 & 1.318 & $0.846-2.053$ \\
\hline$\geq 15$ years & 0.843 & 0.256 & 10.838 & $0.001^{*}$ & 2.324 & $1.407-3.841$ \\
\hline \multicolumn{7}{|c|}{ Treatment measures (control group: diet + exercise) } \\
\hline HD + insulin & 1.803 & 0.519 & 12.078 & $0.001^{*}$ & 6.07 & $2.195-16.785$ \\
\hline \multicolumn{7}{|c|}{ DPN (control group: without DPN) } \\
\hline With DPN & 1.113 & 0.386 & 8.322 & $0.004^{*}$ & 3.042 & $1.429-6.479$ \\
\hline Unclear & 0.323 & 0.191 & 2.843 & 0.092 & 1.381 & $0.949-2.01$ \\
\hline \multicolumn{7}{|l|}{ VTDR } \\
\hline Constant & -6.217 & 1.079 & & & & \\
\hline \multicolumn{7}{|c|}{ Course of disease (control group: $<5$ years) } \\
\hline $5-14$ years & 1.587 & 1.116 & 2.023 & 0.155 & 4.89 & $0.549-43.583$ \\
\hline$\geq 15$ years & 3.163 & 1.071 & 8.725 & $0.003^{*}$ & 23.645 & $2.899-192.874$ \\
\hline
\end{tabular}

* indicate the difference is significant. DR, diabetic retinopathy; HD, hypoglycemic drug; DPN, diabetic peripheral neuropathy; VTDR, vision-threatening diabetic retinopathy; SE, standard error; Sig, significance; Exp, exponential.

diabetic patients based on risk has been shown to be costeffective (21). and can help to reduce the economic burden of fundus screening. We can use the database to develop deep learning or artificial intelligence model and predict the risk of DR progress. On this basis, personalized screening and intervention can be exerted.

Diabetes medication had a greater impact on the prevalence of DR than the other factors, and diabetic patients treated only with diet and exercise had the lowest prevalence of DR. The regression analysis showed that, compared to patients treated only with diet and exercise (i.e., no medication), the prevalence of DR was 6.07 times higher $(\mathrm{P}=0.001)$ in those treated with HDs plus insulin, and 3.334 times higher $(\mathrm{P}=0.011)$ in those treated with oral HDs only. The prevalence of DR was not significantly different between patients treated with insulin injections only and those treated with diet and exercise only. However, Song et al.'s meta-analysis showed that the prevalence of DR was higher in insulin-treated patients than in noninsulin-treated patients (OR $=1.99,95 \%$ CI: 1.34-2.95) (15), and Kumari et $a l$. and Zhao et al. also reported a higher prevalence of DR in insulin-treated patients $(17,22,23)$. A growing number 
of studies have also shown that hypoglycemics including incretins, such as glucagon-like peptide-1 receptor agonists (GLP-1) and sodium/glucose cotransporter-2 inhibitors (SGLT2i), are associated with the suppression of DR occurrence and progression $(24,25)$. Therefore, differences in DR prevention using specific therapeutic measures should be further validated in cohort studies and controlled clinical trials.

In addition, the analysis showed that the incidence of DR in diabetic patients covered with basic health insurance was lower than that of diabetic patients without health insurance, highlighting the reducing effect of basic health insurance policies on the occurrence of DR. These findings suggest that Chinese policy makers should further increase or improve basic health insurance coverage and guarantees, and that standardized and reasonable chronic disease management and guidance should be given to diabetic patients.

The present study has some limitations that should be noted. This is a cross-sectional study, and therefore, the causal relationship between risk factors and DR cannot be analyzed. A case-control or cohort study should be carried out for further analysis. Also, the limited variety of clinical biological indicators in diabetic patients may affect the determination of relevant risk factors. Other risk factors (such as elevated glycated hemoglobin levels, smoking, drinking, BMI, and dyslipidemia) reported in previous studies were not found to be associated with DR or VTDR in our study. Because of the limited number of cases collected by this epidemiological study and incomplete data on patients provided by the Centre, we can't analyze the relevant factors, which is the next step we need to do. Therefore, future community surveys should further expand the relevant biological indicators to obtain more comprehensive conclusions.

\section{Conclusions}

This study investigated the current status of the occurrence of retinopathy and related risk factors in diabetic patients in the communities of Shenzhen in the southern part of China. The results showed that the occurrence of retinopathy in diabetic patients in the southern part of China is high, which is consistent with that in the eastern part of China. The potential reasons are that on the one hand, the prevalence of DM is high, and the disease progression leads to the high prevalence of DR; on the other hand, blood glucose control, diet and exercise management have not yet reached the ideal level, so the incidence of DR is high. To reduce the incidence of DR, we should implement standardized management of DM patients, strengthen the assistance of clinicians and nursing staff to formulate personalized nursing plan, and carry out health education on medication, diet, exercise and psychology. To reduce the incidence of DR, we should implement standardized management of DM patients, strengthen the assistance of clinicians and nursing staff to formulate personalized nursing plan, and carry out health education on medication, diet, exercise and psychology. Disease duration, type of health insurance, medication mode, and the occurrence of DPN are risk factors for the occurrence of DR. Health education regarding retinopathy prevention for diabetic patients, further verification and expansion of health insurance coverage, increased monitoring of glycosylated hemoglobin and diabetic complications, improved fundus monitoring for diabetic patients through digital retinal exams without dilation, and remote screening should be promoted as key measures for DR prevention in the southern part of China.

\section{Acknowledgments}

Funding: The present study was supported by Shenzhen Clinical Research Project (NO. SZLY2017026) and the Shenzhen Sanming Project (NO. szsm201512039).

\section{Footnote}

Reporting Checklist: The authors have completed the MDAR checklist. Available at http://dx.doi.org/10.21037/apm-202526

Data Sharing Statement: Available at http://dx.doi. org/10.21037/apm-20-2526

Conflicts of Interest: All authors have completed the ICMJE uniform disclosure form (available at http://dx.doi. org/10.21037/apm-20-2526). The authors have no conflicts of interest to declare.

Ethical Statement: The authors are accountable for all aspects of the work in ensuring that questions related to the accuracy or integrity of any part of the work are appropriately investigated and resolved. This study was approved by the Ethics Committee of Shenzhen Eye Hospital (No. 2017041201), and informed consent was 
obtained from all diabetic patients who participated in the screening. The study was conducted in accordance with the Declaration of Helsinki (as revised in 2013).

Open Access Statement: This is an Open Access article distributed in accordance with the Creative Commons Attribution-NonCommercial-NoDerivs 4.0 International License (CC BY-NC-ND 4.0), which permits the noncommercial replication and distribution of the article with the strict proviso that no changes or edits are made and the original work is properly cited (including links to both the formal publication through the relevant DOI and the license). See: https://creativecommons.org/licenses/by-nc-nd/4.0/.

\section{References}

1. Hou Q LY, Li S, et al. The global burden of diabetes mellitus. Chinese Journal of Diabetes 2016;1:92-6.

2. Yau JW, Rogers SL, Kawasaki R, et al. Global prevalence and major risk factors of diabetic retinopathy. Diabetes Care 2012;35:556-64.

3. Wong TY, Sabanayagam C. The War on Diabetic Retinopathy: Where Are We Now? Asia Pac J Ophthalmol (Phila) 2019;8:448-56.

4. Zhang X, Saaddine JB, Chou CF, et al. Prevalence of diabetic retinopathy in the United States, 2005-2008. JAMA 2010;304:649-56.

5. Jones CD, Greenwood RH, Misra A, et al. Incidence and progression of diabetic retinopathy during 17 years of a population-based screening program in England. Diabetes Care 2012;35:592-6.

6. Lee R, Wong TY, Sabanayagam C. Epidemiology of diabetic retinopathy, diabetic macular edema and related vision loss. Eye Vis (Lond) 2015;2:17.

7. Thomas RL, Dunstan FD, Luzio SD, et al. Prevalence of diabetic retinopathy within a national diabetic retinopathy screening service. Br J Ophthalmol 2015;99:64-8.

8. Xu J, Xu L, Wang YX, et al. Ten-year cumulative incidence of diabetic retinopathy. The Beijing Eye Study 2001/2011. PLoS One 2014;9:e111320.

9. Jin P, Peng J, Zou H, et al. The 5-year onset and regression of diabetic retinopathy in Chinese type 2 diabetes patients. PLoS One 2014;9:e113359.

10. Dutra Medeiros M, Mesquita E, Papoila AL, et al. First diabetic retinopathy prevalence study in Portugal: RETINODIAB Study--evaluation of the screening programme for Lisbon and Tagus Valley region. $\mathrm{Br} \mathrm{J}$ Ophthalmol 2015;99:1328-33.
11. Klein BE. Overview of epidemiologic studies of diabetic retinopathy. Ophthalmic Epidemiol 2007;14:179-83.

12. Shaw JE, Sicree RA, Zimmet PZ. Global estimates of the prevalence of diabetes for 2010 and 2030. Diabetes Res Clin Pract 2010;87:4-14.

13. Wilkinson CP, Ferris FL, 3rd, Klein RE, et al. Proposed international clinical diabetic retinopathy and diabetic macular edema disease severity scales. Ophthalmology 2003;110:1677-82.

14. Pan CW, Wang S, Qian DJ, et al. Prevalence, Awareness, and Risk Factors of Diabetic Retinopathy among Adults with Known Type 2 Diabetes Mellitus in an Urban Community in China. Ophthalmic Epidemiol 2017;24:188-94.

15. Song P, Yu J, Chan KY, et al. Prevalence, risk factors and burden of diabetic retinopathy in China: a systematic review and meta-analysis. J Glob Health 2018;8:010803.

16. Xu J, Wei WB, Yuan MX, et al. Prevalence and risk factors for diabetic retinopathy: the Beijing Communities Diabetes Study 6. Retina 2012;32:322-9.

17. Varma R, Wen G, Jiang X, et al. Prevalence of Diabetic Retinopathy in Adult Chinese American Individuals: The Chinese American Eye Study. JAMA Ophthalmol 2016;134:563-9.

18. Dutra Medeiros M, Mesquita E, Gardete-Correia L, et al. First Incidence and Progression Study for Diabetic Retinopathy in Portugal, the RETINODIAB Study: Evaluation of the Screening Program for Lisbon Region. Ophthalmology 2015;122:2473-81.

19. Wat N, Wong RL, Wong IY. Associations between diabetic retinopathy and systemic risk factors. Hong Kong Med J 2016;22:589-99.

20. Raum P, Lamparter J, Ponto KA, et al. Correction: Prevalence and Cardiovascular Associations of Diabetic Retinopathy and Maculopathy: Results from the Gutenberg Health Study. PLoS One 2015;10:e0139527.

21. Vujosevic S, Aldington SJ, Silva P, et al. Screening for diabetic retinopathy: new perspectives and challenges. Lancet Diabetes Endocrinol 2020;8:337-47.

22. Kumari N, Bhargava M, Nguyen DQ, et al. Six-year incidence and progression of diabetic retinopathy in Indian adults: the Singapore Indian Eye study. Br J Ophthalmol 2019;103:1732-9.

23. Zhao C, Wang $\mathrm{W}, \mathrm{Xu} \mathrm{D}$, et al. Insulin and risk of diabetic retinopathy in patients with type 2 diabetes mellitus: data from a meta-analysis of seven cohort studies. Diagn Pathol 2014;9:130.

24. Chung YW, Lee JH, Lee JY, et al. The Anti-Inflammatory 
Effects of Glucagon-Like Peptide Receptor Agonist

Lixisenatide on the Retinal Nuclear and Nerve Fiber

Layers in an Animal Model of Early Type 2 Diabetes. Am J Pathol 2020;190:1080-94.

25. Chung YR, Ha KH, Lee K, et al. Effects of sodium-glucose cotransporter-2 inhibitors and dipeptidyl peptidase-4 inhibitors on diabetic retinopathy and its progression: A real-world Korean study. PLoS One 2019;14:e0224549.

(English Language Editor: A. Kassem)

Cite this article as: Peng Y, Guo Q, Liu J, Yao Y, Guo H, Yang M, Wang Y, Xiao X, Ye L. Incidence and risk factors for diabetic retinopathy in the communities of Shenzhen. Ann Palliat Med 2021;10(1):615-624. doi: 10.21037/apm-20-2526 